\title{
BMJ Open Barriers to shared decision-making with women of reproductive age affected by a chronic inflammatory disease: a mixed- methods needs assessment of dermatologists and rheumatologists
}

\author{
Suzanne Murray (10 , ${ }^{1}$ Monica Augustyniak (1) , ${ }^{1}$ Jenny E Murase, ${ }^{2,3}$ \\ Rebecca Fischer-Betz, ${ }^{4}$ Catherine Nelson-Piercy, ${ }^{5}$ Morgan Peniuta, ${ }^{1}$ Ivo Vlaev (i) ${ }^{6}$
}

To cite: Murray S,

Augustyniak M, Murase JE, et al. Barriers to shared decision-making with women of reproductive age affected by a chronic inflammatory disease: a mixed-methods needs assessment of dermatologists and rheumatologists. BMJ Open 2021;11:e043960. doi:10.1136/ bmjopen-2020-043960

- Prepublication history and additional supplemental material for this paper are available online. To view these files, please visit the journal online (http://dx.doi.org/10.1136/ bmjopen-2020-043960).

Received 21 August 2020 Accepted 18 May 2021

Check for updates

(C) Author(s) (or their employer(s)) 2021. Re-use permitted under CC BY-NC. No commercial re-use. See rights and permissions. Published by BMJ.

For numbered affiliations see end of article.

Correspondence to

Ms Suzanne Murray; murrays@axdevgroup.com

\section{ABSTRACT}

Objectives The main study objective was to identify challenges and barriers experienced by dermatologists and rheumatologists when engaging women of reproductive age in shared decision-making (SDM) related to treatment and management of chronic inflammatory disease (CID) before, during and after pregnancy.

Design A mixed-methods study was conducted, employing (1) semistructured interviews, (2) an online survey and (3) triangulation of findings.

Participants 524 dermatologists and rheumatologists entered the study; 495 completed it; 388 met inclusion criteria for analysis. Participants were included if actively practising in Germany (GER), the UK or the USA; had a minimum $5 \%$ caseload of female patients of reproductive age with either axial spondyloarthritis, psoriasis, psoriatic arthritis or rheumatoid arthritis; and had experience prescribing biologics.

Results 48 interviews and 340 surveys were analysed. Interviews underscored dermatologists and rheumatologists' suboptimal integration of SDM in clinical practice. In the survey, $90 \%(n=305)$ did not know about SDM models. A perceived lack of competency counselling patients on pregnancy and family planning was also identified during interviews. Among the survey sample, $44 \%$ ( $n=150)$ of specialists agreed they preferred leaving pregnancy-related discussions to obstetricians and/ or gynaecologists and $57 \%(n=189)$ reported having suboptimal skills discussing contraceptive methods with patients. Another finding that emerged from interviews was the perception that all biologics are strictly contraindicated during pregnancy. Suboptimal knowledge was noted among $57 \%(n=95)$ of dermatologists and $48 \%(n=83)$ of rheumatologists surveyed in that regard, with a statistically significant difference by country among dermatologists (GER: $42 \%$ vs UK: $71 \%$ vs USA: $57 \%, p=0.015$ ).

Conclusions This study identified low levels of knowledge, skill and confidence, as well as attitudinal issues, that explain why SDM is not fully integrated in dermatology and rheumatology clinical practice. Blendedlearning interventions are recommended to assist CID specialists in developing effective communication and patient engagement competencies.
Strengths and limitations of this study

- To the coauthors' knowledge, this is the first needs assessment that captures dermatologists' and rheumatologists' perspectives on the topic of shared decision-making with women of reproductive age affected by a chronic inflammatory disease.

- A purposive sampling approach was used to ensure that a wide spectrum of experiences was considered within the examined population group.

- A triangulation of multiple sources of data, methods and perspectives was conducted to minimise singleobserver and single-methods biases.

- Results of this study should not be generalised to all professions within the field of chronic inflammatory disease, nor to countries or health systems outside those included in the study.

- The knowledge, skills, confidence and attitudes of dermatologists and rheumatologists were self-reported and not tested through validated instruments.

\section{INTRODUCTION}

Physicians face challenges when treating and managing women of reproductive age with a chronic inflammatory disease (CID), ${ }^{1}$ such as axial spondyloarthritis (axSpA), psoriatic arthritis (PsA), psoriasis ( $\mathrm{PsO}$ ) and rheumatoid arthritis (RA). Special consideration is required regarding the (a) impact of a potential pregnancy on disease activity, symptoms or long-term damage to joints and organs, ${ }^{2}$ (b) impact of disease activity on pregnancy and childbirth (eg, growth restrictions and preterm delivery), ${ }^{34}$ and (c) the impact of treatment on both maternal and fetal health. ${ }^{4-7}$ Agents used to treat CID subtypes include classical disease-modifying drugs (eg, immunosuppressants, retinoids and corticosteroids), targeted therapies (eg, Januse kinase (JAK) and phosphodiesterase-4 
(PDE4) inhibitors) and biologics (eg, tumour necrosis factor (TNF) inhibitors and interleukin inhibitors). Evidence and recommendations regarding the use of available agents before, during and after pregnancy vary across guidelines. ${ }^{5-10}$

Some disease-modifying agents (eg, methotrexate, leflunomide and acitretin) are contraindicated during pregnancy due to teratogenic effects. ${ }^{5-7}$ Others (eg, hydroxychloroquine, sulfasalazine, azathioprine and ciclosporin) are ineffective for axSpA and severe RA. ${ }^{7}$ Evidence on pregnancy and childbirth outcomes associated with JAK, PDE4 and interleukin inhibitors is insufficient. ${ }^{59}$ Among TNF inhibitors, certolizumab pegol is the only therapeutic option with sufficient evidence of negligible placental transfer ${ }^{11}{ }^{12}$ recommended by the British Association of Dermatologists as first-line biologic during conception $^{9}$ and continuation throughout pregnancy by the European League Against Rheumatism and the American College of Rheumatologists. ${ }^{57}$ Administration of other TNF blocking agents during the third trimester of pregnancy can expose the neonate to significant drug levels. ${ }^{.13}$

Shared decision-making (SDM) can be used to address uncertain decisions. ${ }^{14-19}$ The end goal is for the physician and patient to share the responsibility of a selected treatment and management option. This is based on a discussion of the patient's preferences and values, as well as the physician's clinical expertise and application of evidencebased medicine. ${ }^{151720} \mathrm{SDM}$ is not implemented in clinical practice as much as it is advocated for. Across 168 consultations with patients affected with RA, the average score of SDM observed among rheumatologists was 28 on the observer patient involvement (OPTION) scale (0-100), indicating a low level of involvement in the process. ${ }^{21}$ In another study conducted in the Netherlands, dermatologists had a significantly higher perception of SDM engagement (95\% CI 80 to 83 ) compared with patients with atopic dermatitis or $\mathrm{PsO}$ (95\% CI 51 to 58, p<0.01), indicating a discrepancy in SDM involvement. ${ }^{22}$ Both studies suggest potential barriers and challenges to engaging patients in SDM. Therefore, the objectives of this study were to:

1. Assess the knowledge, clinical decision-making skills and confidence of dermatologists and rheumatologists in the areas of risk assessment, treatment and management of women of reproductive age with CID.

2. Assess the interprofessional communication and collaboration skills of healthcare professionals (HCPs) and their perspectives on SDM with patients.

3. Identify challenging tasks, situations and barriers (obstacles) preventing optimal decision-making with female patients who have CID before, during or after pregnancy.

4. Inform continuous professional development activities and performance improvement interventions in the fields of dermatology, rheumatology and patient-provider communication.

\section{METHODS}

\section{Study design}

A mixed-methods educational and behavioural needs assessment was conducted including two data collection and analysis phases (qualitative followed by quantitative). ${ }^{23-25}$ The qualitative phase involved semistructured interviews with dermatologists and rheumatologists to explore meaning, context and underlying causalities of self-reported barriers and challenges encountered in clinical practice. The targeted number of interviews was determined a priori based on expected requirements to reach data saturation and maximum variation in purposive sampling criteria. ${ }^{26}$ Qualitative findings informed the development of an online survey to assess the magnitude and frequency of self-reported barriers and challenges in a larger sample of dermatologists and rheumatologists. The sample size for the survey was calculated to reach a statistical power of 0.8 with $\alpha=0.05$ and a large effect size (Cohen's w=0.5) for $6 \times 2 \mathrm{X}^{2}$ tests. $^{27}$ All sources of information, data analyses and points of interpretation were triangulated to obtain an in-depth understanding of the challenges and barriers identified, as well as their underlying causalities. ${ }^{25}$ This approach has been effectively used in previous studies to identify educational needs of $\mathrm{HCPs}^{28-30}$ and helps minimise bias. ${ }^{25}$

\section{Theoretical framework}

A discrepancy model was used to assess gaps between 'what is' and 'what should be ${ }^{24}$ via validated constructs of behaviour change relevant to medical education and training. ${ }^{31}$ The following components of the Capability, Opportunity, Motivation, Behaviour (COM-B) model and theoretical domains framework were applied: psychological capability (knowledge), physical capability (skills), reflective motivation (attitude and confidence) and social opportunity (interprofessional communication and collaboration, including team dynamics and perceived social norms), ${ }^{31-33}$ in the context of a system and a practice that requires both individual and interprofessional competencies. ${ }^{34}$ Various models and perspectives of SDM were used in the collection and analysis of data. ${ }^{14-18} 2035$

\section{Recruitment}

Participants were recruited from two separate international online panels of HCPs in compliance with the European society for opinion and marketing research. ${ }^{36}$ Invitation letters were sent by email, introducing potential participants to the study. A secured URL directed interested participants to an online screener and consent form. Eligible participants were directed to an availability form (qualitative phase) or to the online survey (quantitative phase).

\section{Research criteria}

Participants were deemed eligible for the study if they: (1) practised in Germany (GER), the UK or the USA; (2) were certified dermatologists or rheumatologists; (3) were actively practising (ie, $\geq 50 \%$ of time is spent 
caring for patients, primary practice is not researching or teaching and they are not retired); (4) had at least 3 years of clinical practice; (5) had a minimum caseload (5\%) of female patients of reproductive age with either axSpA, PsA, PsO or RA; and (6) had some experience prescribing biologics for the treatment of these conditions. Purposive sampling was applied to include participants with different years of practice, locations (ie, urban, suburban or rural), settings (ie, academic affiliated or community based) and gender. ${ }^{37}$

\section{Data collection}

\section{Qualitative phase: semistructured interviews}

Semistructured interview guides were developed in English based on predetermined areas of exploration, ${ }^{38}$ which were identified via literature review including published models and perspectives of SDM and behaviour change. The final interview guide was then translated into German and verified by a German to English translator for quality assurance. Expert interviewers (MA and MP) trained additional professional qualitative interviewers with a background in healthcare to ensure alignment with study objectives, questions and interviewing techniques. Each interview was completed over a conference call, recorded on participant's consent and lasted $45 \mathrm{~min}$. Audio recordings were transcribed. German transcripts were translated to English. Details on the interview guide can be found in online supplemental file 1 .

\section{Quantitative phase: online survey}

A 15-minute survey consisting of 16 questions was developed based on qualitative findings and the literature. Knowledge and skills were rated using a 5-point scale: (1) none, (2) basic, (3) intermediate, (4) advanced and (5) expert. Confidence was rated on a $0 \%$ (low confidence) to $100 \%$ (high confidence) visual analogue scale.
Participants' attitude was assessed via a 5-point agreement scale: (1) strongly disagree, (2) disagree, (3) neither agree nor disagree, (4) agree and (5) strongly agree. For any given item that required a rating, the option 'not relevant to my current role' was provided. The survey was reviewed for content and face validity by coauthors with clinical, behavioural and educational expertise (SM, JEM, RF, CN-P, IV), and then it was translated to German and verified by a German to English translator for quality assurance. Final English and German versions were programmed online and beta-tested to ensure optimal navigation. Three validation questions, asking participants to select a specific answer, were included to monitor participant's attention to item content. ${ }^{39}$ 'Careless respondents' (ie, participants who responded without regard to item content ${ }^{40}$ ) were excluded from analysis (see figure 1 for further explanation). Details on the survey can be found in online supplemental file 1 .

\section{Data analysis \\ Qualitative analysis}

Transcripts were transferred into NVivo V.12 (QSR International) and analysed using a hybrid method that combined both an inductive and deductive approach. ${ }^{41}$ The coding tree was composed of defined categories covering all predetermined areas of exploration and was refined as more transcripts were read and analysed for emergent themes (online supplemental file 2).$^{42}$ Matrices were then developed to analyse themes by subgroup (eg, specialty) ${ }^{43}$ For each emerging theme, one or two representative quotes were selected.

\section{Quantitative analysis}

Final survey data were transferred into a database (IBM SPSS Statistics for Windows, V.25.0) and cleaned to remove incomplete or duplicate cases. Variables that
Qualitative Phase: Semi-Structured Interviews

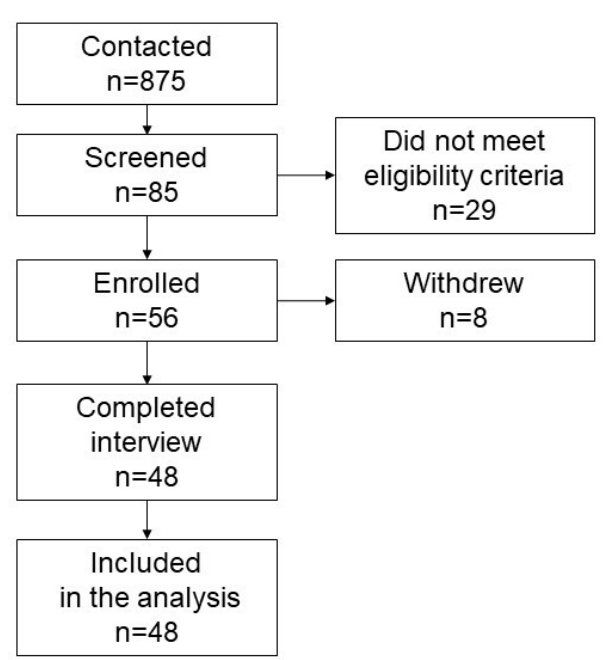

Quantitative Phase: Online Survey

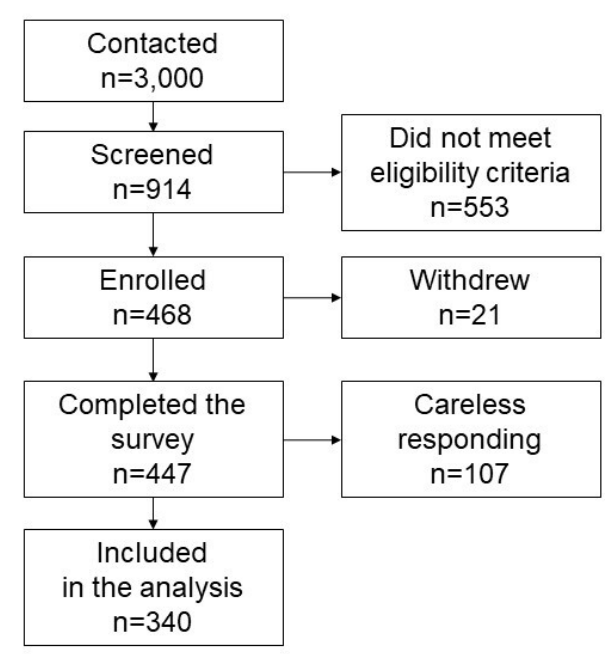

Figure 1 Recruitment of participants leading to final samples of qualitative and quantitative phases. Three validation questions were included sporadically throughout the survey with concrete instructions for the participant to select a specific response. Participants were considered 'careless' if they failed at least two attention-validation questions $(n=107)$. 
reflected a knowledge or skill rating were regrouped as follows: suboptimal if (1) none, (2) basic or (3) intermediate; and optimal if (4) advanced or (5) expert. Variables that reflected an agreement rating were regrouped as follows: disagree if (1) strongly disagree or (2) disagree; neutral if (3) neither agree nor disagree; and agree if (4) agree or (5) strongly agree. Participants who selected 'not relevant to my current role' were excluded from singleitem analysis. Frequency tables were created for analysis of demographics; cross-tabulations between dependent variables (knowledge, skill or agreement items) and independent variables (specialty, country), along with $\mathrm{X}^{2}$ tests with $\alpha=0.05$ were created for subgroup analysis. KruskalWallis $\mathrm{H}$ tests, a non-parametric test that assigns a rank based on the value of a measured score, were used to assess statistically significant differences in mean rankings of confidence scores between subgroups. ${ }^{44}$ Therefore, lower ranks indicate lower confidence in a respective subgroup. This method has been used by previous studies in the field of behavioural and educational research specialised in healthcare. ${ }^{29} 30$

\section{Triangulation}

Data from qualitative interviews and quantitative surveys were compared to identify areas of convergence or divergence. ${ }^{25}$ The meaning and potential causalities of identified barriers and challenges were discussed among coauthors.

\section{Patient involvement}

This study examines the barriers to SDM from the perspective of dermatologists and rheumatologists. Due to the scope of this investigation, patients were not involved.

\section{RESULTS}

A total of 48 interviews and 340 surveys were analysed (figure 1). The study population consisted mostly of male dermatologists and rheumatologists $(\mathrm{n}=239,62 \%)$ with $11-20$ years of practice $(n=202,52 \%)$. German $(n=102$, $77 \%)$ and UK physicians $(\mathrm{n}=70,58 \%)$ worked mostly in urban locations, meanwhile those in the USA practised mostly in suburban locations $(\mathrm{n}=77,57 \%)$. The highest mean caseload of patients reported by dermatologists, in their practice, was those affected with $\mathrm{PsO}: 36 \%( \pm 25 \%)$ in GER, $27 \%( \pm 19 \%)$ in the UK and $18 \%( \pm 16 \%)$ in the USA. Meanwhile, for rheumatologists the highest caseloads tended to be patients with RA, reaching 30\% $( \pm 21 \%)$ in GER, $30 \%( \pm 18 \%)$ in the UK and $32 \%( \pm 23 \%)$ in the USA. Most participants $(n=292,86 \%)$ reported prescribing biologics often for the treatment of axSpA, PsA, PsO or RA (table 1).

\section{Main findings}

Four findings emerged from the data for challenges and barriers: (1) selecting an optimal treatment choice with women of reproductive age, (2) discussing childbearing aspirations and managing unplanned pregnancy, (3)

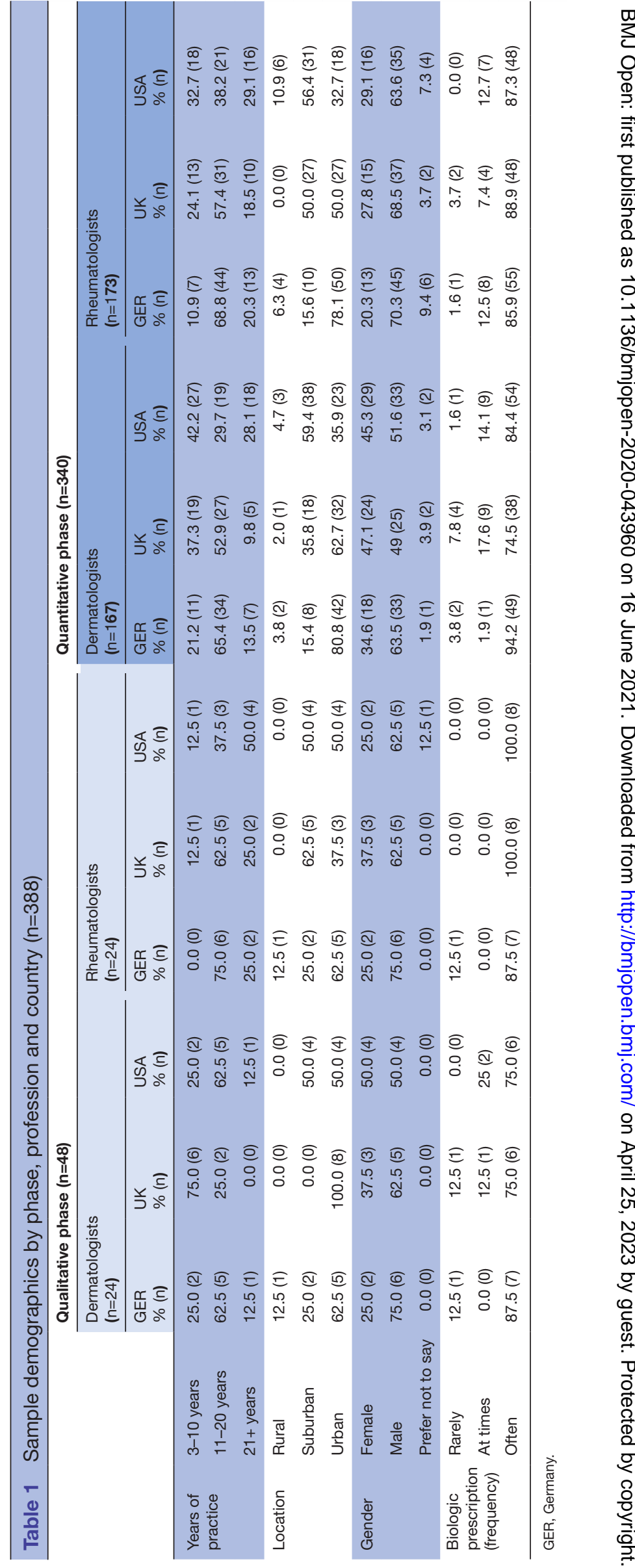


integrating SDM into clinical practice, and (4) collaborating with obstetricians and/or gynaecologists $(\mathrm{OB} /$ GYNs) and general practitioners (GPs). The findings are prioritised by urgency of need and potential impact of gap mitigation.

\section{Selecting an optimal treatment choice with women of reproductive age}

There was a common generalisation that if a woman with CID becomes pregnant or is at risk of having an unplanned pregnancy, biologics should be discontinued for safety concerns. Participants described having liability concerns due to limited clinical evidence and limited experience:

I think the challenges for biologics are that the evidence is so limited at the moment for patients who are pregnant. It's so nerve-wracking prescribing a biologic to a pregnant woman because it's not something I have done a huge amount of or that my colleagues have done a huge amount of. And then trying to reassure patients about something you're not $100 \%$ comfortable with yourself, that's a challenge. (Dermatologist, UK)

I think there's always uncertainty about the role of drugs and safety of drugs, particularly in pregnancy, as there's no real clinical trials that accept patients who are pregnant or breastfeeding $[\ldots]$ sometimes you're dealing with all that uncertainty and anxiety in patients who have suddenly got pregnant without it being planned. (Rheumatologist, UK)

Discontinuation of biologics during pregnancy paralleled a perceived lack of knowledge regarding the impact of CID on women's reproductive health (table 2, items 1 and 2). Similar trends were noticed for suboptimal knowledge of available data regarding the compatibility of biologic injectables (eg, TNF and interleukin inhibitors) during pregnancy and lactation (see table 2, items 3-6). Participants' confidence prescribing biologic injectables was lower during pregnancy (average 65\%, $\mathrm{n}=336$ ) compared with before or after pregnancy (average $75 \%$, $\mathrm{n}=338$ ). In both cases of biologic prescription, dermatologists in the UK had the lowest mean confidence score (figure 2, items 1 and 2).

\section{Discussing childbearing aspirations and managing unplanned pregnancies}

Participants reported having limited skills discussing contraceptive methods with patients (table 2, item 7 ) and limited confidence to perform this task (average 60\%, $\mathrm{n}=318$ ). The lowest mean confidence score ranks were obtained among rheumatologists in the UK and GER (figure 2, item 3). Almost half of participants (44\%, 150 of 338) indicated that they preferred leaving pregnancyrelated discussions to $\mathrm{OB} / \mathrm{GYNs}$ (table 3, item 4). The quote below illustrates a perceived lack of competency:

...I may briefly mention how it [discontinuing treatment] may affect the pregnancy in general or the fetus, but [for] most of the discussion specifically about the pregnancy and the baby's development, I tend to defer to obstetricians and high-risk OB-GYN doctors. I feel that that's more in their area of expertise. (Rheumatologist, USA)

Dermatologists and rheumatologists reported suboptimal skills closely monitoring changes in pregnancy status or childbearing aspirations $(64 \%, 211$ of 332; table 2, item 8 ) and making appropriate treatment adjustments in the face of unplanned pregnancy $(53 \%, 179$ of 337; table 2, items 11 and 12). The average confidence adjusting treatment in such circumstances was $62 \%$ $(n=333)$. Dermatologists and rheumatologists in the UK ranked the lowest in mean confidence scores (figure 2, item 4). The quote below further illustrates the perceived challenge of discussing pregnancy and contraceptives:

What do you do if the patient becomes pregnant while being on a system therapy? You discontinue but the damage has probably already been made. And then the question starts whether we need an abortion, with what indication. Also, if it's a very religious woman, no matter which religion, can be very catholic or something else; there I don't do anything because I don't enter any religious discussions at all. (Dermatologist, GER)

\section{Integrating SDM into clinical practice}

Dermatologists and rheumatologists demonstrated a lack of knowledge and skill to engage women of reproductive age in SDM. Only 12\% (20 of 167) of dermatologists and $9 \%$ (15 of 173) of rheumatologists reported being aware of SDM models and guidelines. Thirty-six per cent $(36 \%$, 120 of 338) had suboptimal knowledge of SDM strategies; $38 \%$ (128 of 339) had suboptimal skill assessing patients' desired level of involvement in SDM; 35\% (120 of 338) had suboptimal skill adapting to patients' health literacy level when explaining treatment options and 39\% (131 of 338) had suboptimal skill identifying patients' motive for a certain treatment preference (table 2, items 9-13). Unfavourable attitudes towards SDM were found, including a perceived lack of applicability in clinical context, reluctance and inability to engage patients due to inability to communicate at a similar health literacy level:

Neither the patient nor the GP has the competency to tell me what to do. I decide what to do. If I recommend something I have an idea behind. If they don't want it, they can go to a different dermatologist. (Dermatologist, GER)

...in some patients it may be confusing. It's like you are asking them to become doctors overnight, to understand $[\ldots]$ That can be overwhelming for a patient the way I'm overwhelmed when I go to a mechanic and he talks to me about what's wrong with my car. (Rheumatologist, USA) 


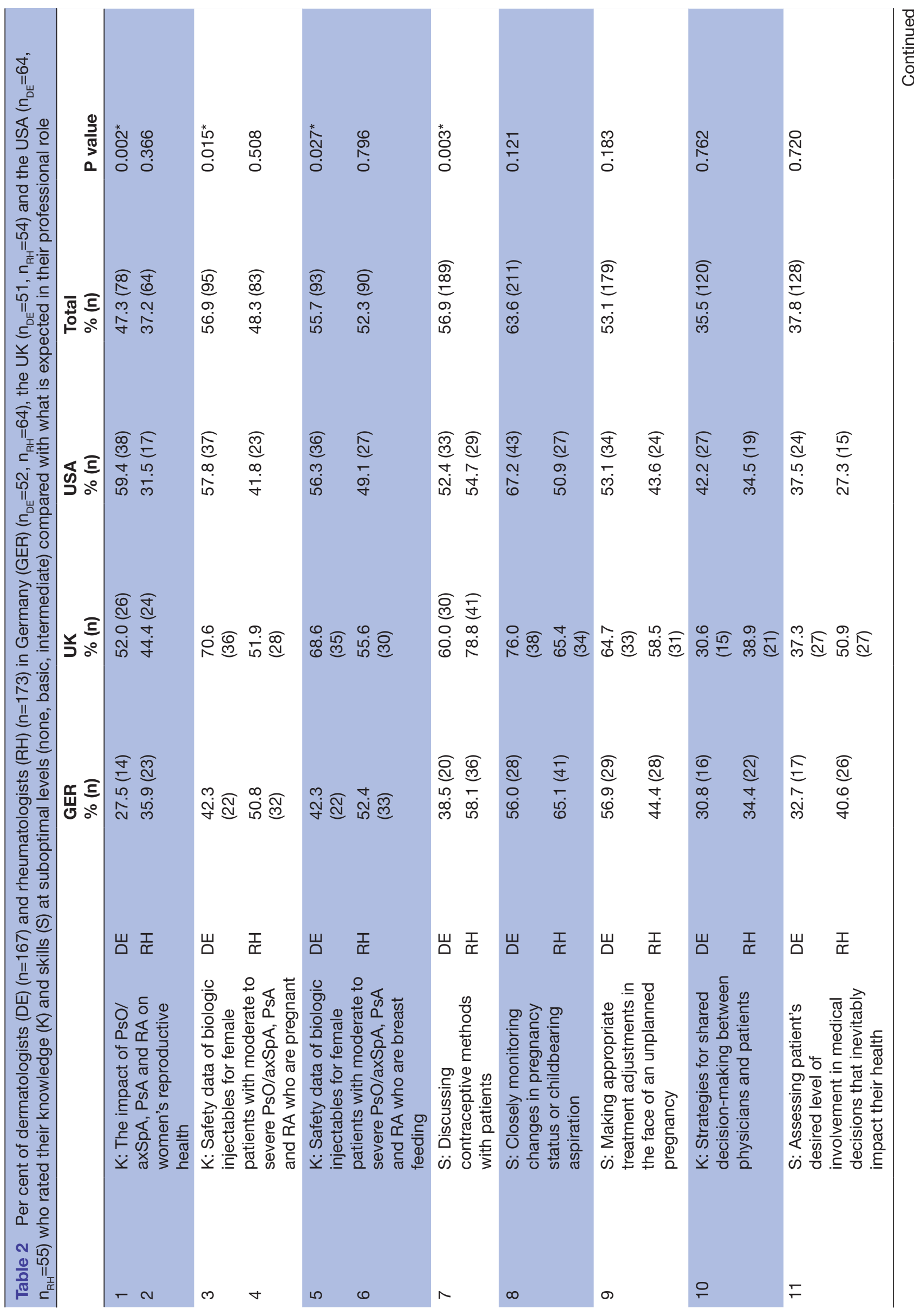




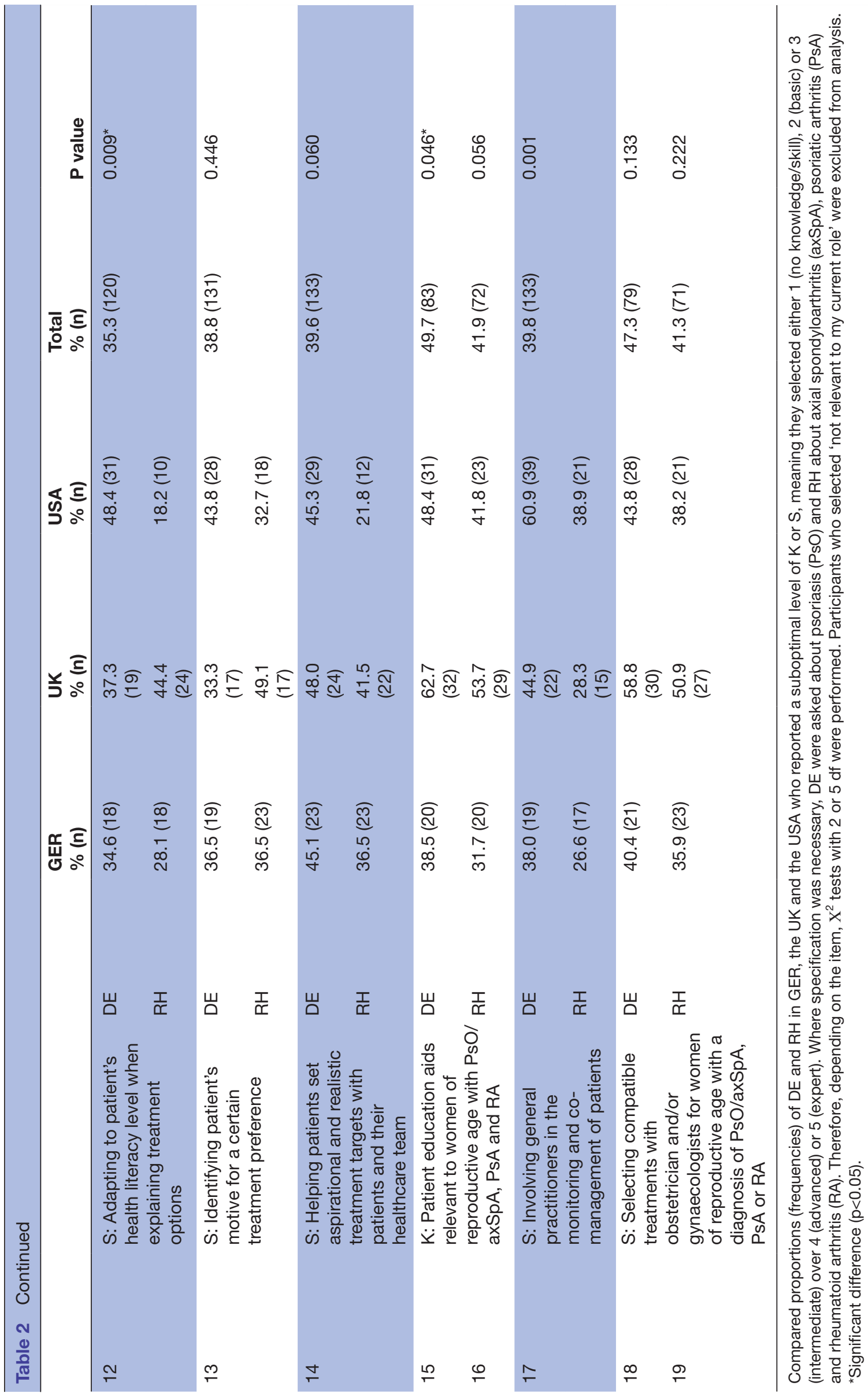


1. Prescribing biologic injectables (e.g. TNF, IL-12, IL-23 and IL-17 inhibitors) before or after pregnancy $(n=338)$

Mean rank confidence score $H(5), 14.497, p=0.013^{*}$

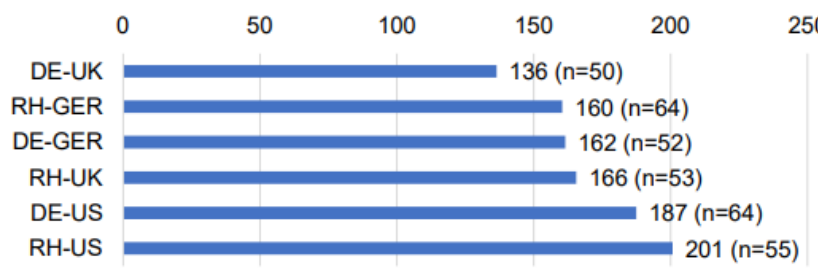

3. Discussing contraceptive methods with patients $(n=318)$

Mean rank confidence score

$H(5), 16.465, p=0.006^{*}$

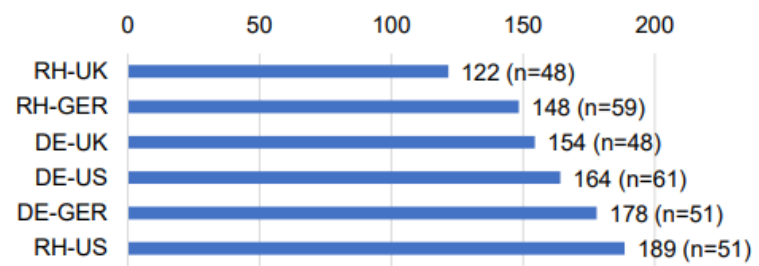

2. Prescribing biologic injectables (e.g. TNF, IL-12, IL-23 and IL-17 inhibitors) during pregnancy $(n=336)$

Mean rank confidence score $\mathrm{H}(5), 28.788, \mathrm{p}<0.001^{*}$

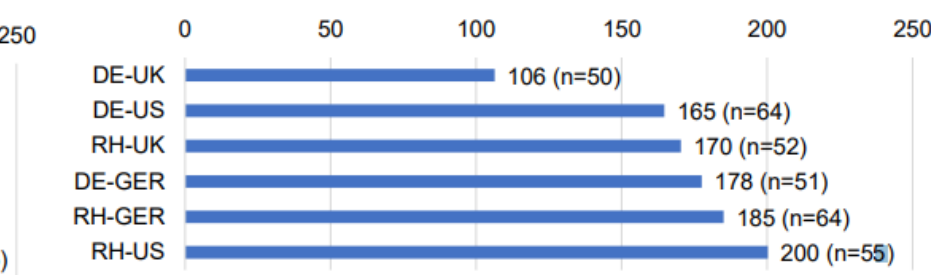

4. Adjusting treatment in the face of unplanned pregnancies $(n=333)$

Mean rank confidence score $H(5), 13.812, p=0.017^{*}$

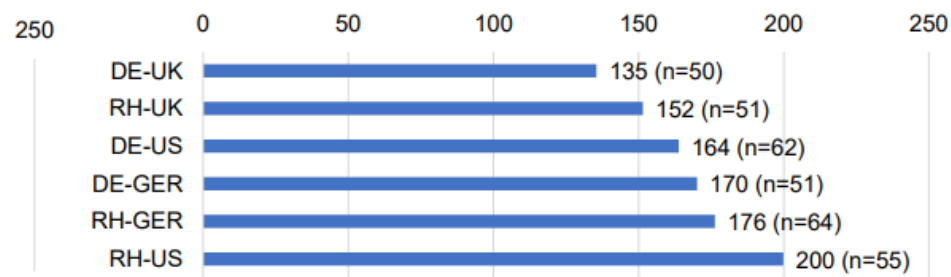

Figure 2 Mean rank scores for confidence of dermatologists $(n=167)$ and rheumatologists $(n=173)$ in Germany $(n D E=52$, $\mathrm{nRH}=64)$, the $\mathrm{UK}(\mathrm{nDE}=51, \mathrm{nRH}=54)$ and the USA (nDE=64, $\mathrm{nRH}=55)$. While participants rated their confidence on a scale of $0 \%-100 \%$, this figure reports Kruskal-Wallis $\mathrm{H}$ mean ranks of confidence scores by subgroups. Lower ranks indicate lower confidence. Participants who selected 'not relevant to my current role' were excluded from analysis. *Significant difference $(p<0.05)$. DE-GER, dermatologists in Germany; DE-UK, dermatologists in the UK; DE-US, dermatologists in the USA; IL, interleukin; RH-GER, rheumatologists in Germany; RH-UK, rheumatologists in the UK; RH-US, rheumatologists in the USA; TNF, tumour necrosis factor.

On average, $22 \%$ (74 of 340 ) of participants agreed that they often forget to engage patients in SDM and 25\% (84 of 340) agreed that they feel pressure to make a treatment decision rapidly without patient's input (table 3, items 5 and 6). Further, dermatologists and rheumatologists reported having limited knowledge of patient education aids relevant to women of reproductive age with a CID (table 2, items 15 and 16). There was a perception that available patient decision aids did not include information pertaining to treatment compatibility during pregnancy and lactation.

\section{Collaborating with OB/GYNs and GPs}

Dermatologists and rheumatologists reported having suboptimal skill involving GPs in the monitoring and co-management of patients (table 2, item 17). Almost half of dermatologists (47\%, 79 of 167) and rheumatologists $(41 \%, 71$ of 172$)$ perceived their skills selecting appropriate treatments with $\mathrm{OB} / \mathrm{GYNs}$ as suboptimal (table 2, items 18 and 19). Many experienced challenges within their interprofessional teams, as well as suboptimal communication channels in the system in which they worked (table 3, items 7 and 8). Specifically, 28\% (101 of 340 ) of dermatologists and rheumatologists agreed that it was challenging to deliver medical advice that aligns with what other HCPs recommend to their patients, a situation that is also illustrated by the following quotes:

...the patient virtually 100 percent of the time is going to listen to the OB/GYN and not to what we have to say... (Dermatologist, USA)

So you start somebody on methotrexate, they go and see their GP, their GP will tell the patient this is a really dangerous and toxic drug. The patient then comes back angry because their GP has told them this, so then you have to undo that damage and explain to them again what you had explained in the first place. (Rheumatologist, UK)

\section{DISCUSSION}

This study identified various challenges and barriers affecting dermatologists' and rheumatologists' ability, reflective motivation and social opportunity to engage in SDM with women of reproductive age affected by a CID before, during and after pregnancy. ${ }^{32}$ Both dermatologists and rheumatologists reported low levels of knowledge regarding the compatibility of treatments during pregnancy and lactation; low levels of skill discussing childbearing aspirations and contraceptives; and a lack of 
Table 3 Per cent of dermatologists (DE) $(n=167)$ and rheumatologists $(R H)(n=173)$ in Germany $(G E R)\left(n_{D E}=52, n_{R H}=64\right)$, the UK $\left(n_{D E}=51, n_{R H}=54\right)$ and the USA $\left(n_{D E}=64, n_{R H}=55\right)$ who reported unfavourable attitudes or experienced challenges

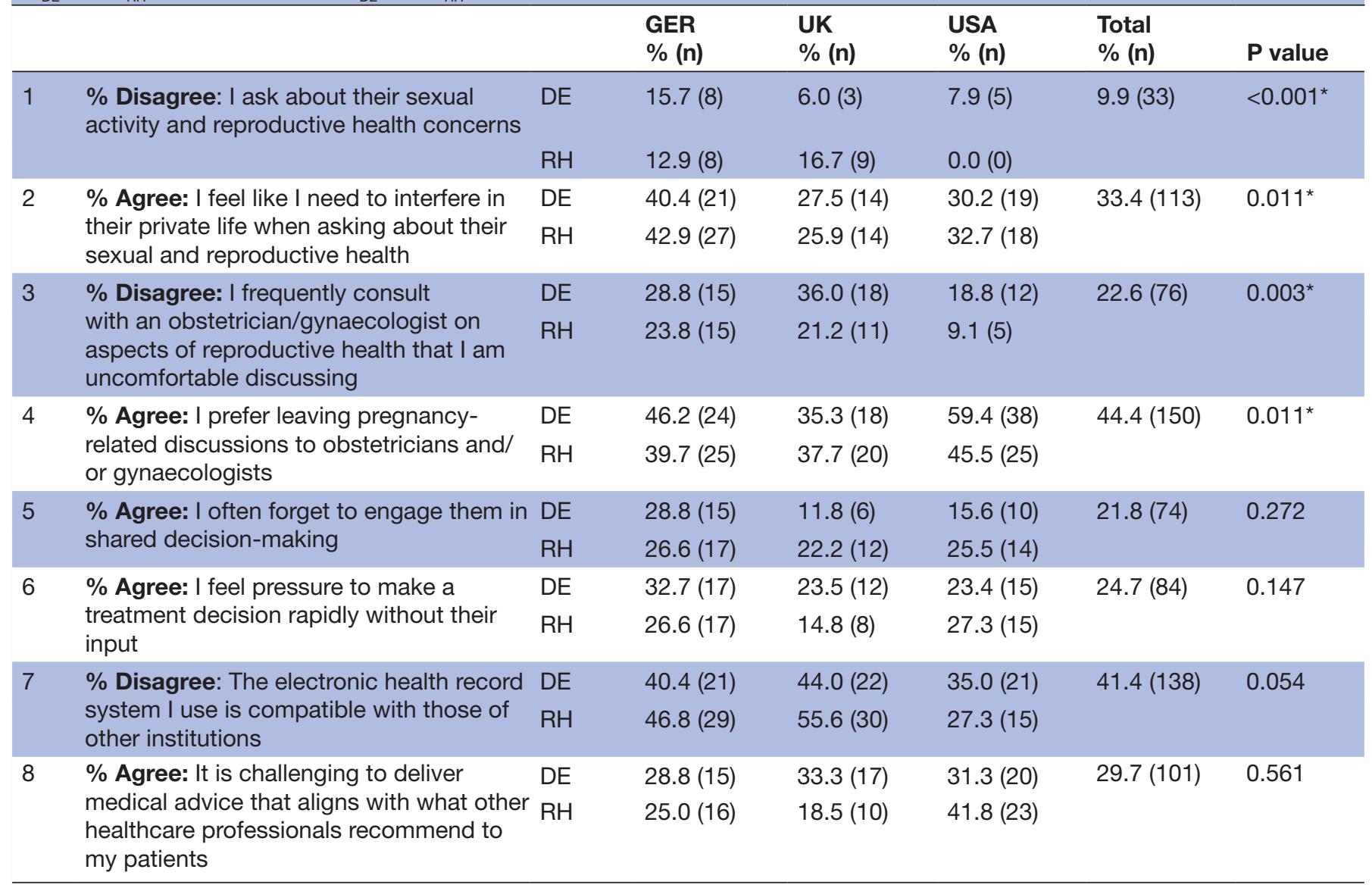

Compared proportions (frequencies) of DE and $\mathrm{RH}$ who selected: (1) agree or strongly agree with statement, or (2) disagree or strongly disagree with statement.

${ }^{*}$ Significant difference $(p<0.05)$.

awareness of SDM models or strategies. Some expressed reluctance to implement SDM in their clinical practice paralleled with low confidence in their patients' ability to engage in this process. Further, dermatologists and rheumatologists reported having to manage misaligned recommendations between themselves, GPs and OB/ GYNs.

\section{Fit within the literature}

A qualitative study previously reported that rheumatologists in the USA are uncertain about their roles and responsibilities in relation to family planning counselling and reproductive healthcare. In addition, they perceive missing guidelines and competing clinical priorities as significant barriers. ${ }^{45}$ The present study suggests that dermatologists and rheumatologists in GER, the UK and the USA may not always consult with OB/GYNs in discussions pertaining to patients' childbearing aspirations in the context of CID treatment. Further, they may lack confidence discussing contraceptives with patients and perceive unplanned pregnancy as a barrier to prescribing biologics. Few factors driving 'clinical inertia' with women of childbearing age affected with
CIDs have been identified in a systematic review. ${ }^{1}$ Factors included clinicians' lack of knowledge of clinical trial data, including pregnancy outcomes and fear of harming the baby. ${ }^{1}$ The present study validates that both dermatologists and rheumatologists have tendencies to discontinue biologics during pregnancy and have suboptimal knowledge of current evidence pertaining to the compatibility of biologics before, during and after pregnancy. Other studies underscored the presence of attitudinal issues pertaining to the implementation of SDM in clinical practice, including perceived time-constraints, ${ }^{46}$ the belief that patients prefer not to engage in $\mathrm{SDM},{ }^{46}$ use of evidence-based medicine being a reason to bypass $\mathrm{SDM}^{47}$ asymmetry in power between physician and patients preventing SDM,$^{46}$ absence of tools, ${ }^{48}$ and perception that SDM is not priority or is already implemented in clinical practice. ${ }^{48}$ The present study suggests that providers may overestimate their implementation of SDM as less than $25 \%$ of participants reported often forgetting to engage in this process or making treatment decisions without patient's input. This study also supports that actual utilisation of SDM may be linked to a lack of knowledge 
regarding current SDM models and strategies to achieve this process in clinical practice in addition to suboptimal communication skills.

\section{Strengths and weaknesses of the study}

To authors' knowledge, this is the first international mixed-methods study presenting the perspective of dermatologists and rheumatologists on the topic of SDM with women of reproductive age affected with a CID. The qualitative phase allowed for new, rich and contextual information, beyond the literature. Meanwhile the quantitative phase helped validate these findings in a larger sample of participants. ${ }^{23}{ }^{25}$ Purposive sampling ensured that a wide spectrum of perspectives was considered within the examined population group (eg, mix of gender). ${ }^{37}$ The triangulation of multiple sources of data, methods and perspectives helped minimise single-observer and single-methods biases. ${ }^{25}$ Attention-validation questions were used to monitor the quality of data collected. ${ }^{40}$ One hundred and seven participants were excluded for failing to follow instructions to at least two attention-validation questions. These questions allowed to minimise inclusion of participants who disregarded item content when answering questions. Survey fatigue may have contributed to the exclusion rate. Findings may not be generalised to all professions working in the field of CID, nor to countries, languages and health systems outside those included in the study. Extensive multivariate analysis to account for more than one confounding variable was not possible. Barriers influencing physical opportunities (eg, policies) and automatic motivation (eg, incentives and rewards) were explored only at a superficial level. ${ }^{32}$ The objective proficiencies and knowledge of dermatologists and rheumatologists were not tested nor captured from the perspective of patients or other HCPs. A self-selection bias may have occurred among dermatologists and rheumatologists with an interest in continuous medical education and/or research.

\section{Implications for clinicians and policymakers}

The present study underscores professional competencies requiring further improvement among dermatologists and rheumatologists in GER, the UK and the USA as it relates to treatment and management discussions with women of reproductive age affected with a CID. These areas for improvement can be addressed through knowledge and skill improvement interventions, ${ }^{31}$ including motivational interviewing as an approach to elicit a reflection and treatment preference by patients. ${ }^{49}$ An additional focus should be placed on addressing attitudinal barriers by connecting learners with expert clinicians or peers to simulate difficult discussions. ${ }^{15}$ For example, Making Good Decisions in Collaboration ${ }^{48}$ engaged clinicians in scenarios that help them recognise discrepancies between current approaches to communication with patients and actual recommendations. Many organisational factors (eg, leadership) and system factors (eg, continuing education policies) have been shown to influence the success and impact of SDM implementation..$^{50} \mathrm{~A}$ recent systematic review of $36 \mathrm{SDM}$ interventions concluded that a wide range of multilevel avenues are feasible, however, more rigorous testing is still required to identify effective approaches. $^{51}$

\section{Unanswered questions and future research}

Future studies may consider exploring the perspective of women of reproductive age with CIDs, as well as GPs and $\mathrm{OB} / \mathrm{GYNs}$. A larger sample size with a wider range of perspectives (eg, ethnicities) would ensure greater implications of results for the disease-affected population. Including the perspective of health administrators and health system policymakers would help investigate challenges affecting clinicians' opportunities and motivations. ${ }^{31}$ In parallel, the application of concepts from behavioural economics and targeting automatic thinking processes in the context of SDM interventions merit further investigation. ${ }^{52}$ Other studies may seek to validate the present barriers and challenges identified among dermatologists and rheumatologists by deploying objective assessments (eg, audiotaped visits). Although recent studies have sought to evaluate the feasibility and impact of implementing SDM in dermatology and rheumatology practice, the heterogeneity of studies prevents systematic reviews from conducting meta-analyses. Longitudinal research on the health outcomes of women of reproductive age exposed to SDM might help uncover this evidence gap. In addition, performance and quality improvement demonstration projects could assess the added value of implementing SDM, while teaching clinicians to overcome challenges and barriers via the use of measurable action plans, such as Plan, Do, Study, Act cycles. ${ }^{53}$ Lastly, further research is required to evaluate the effectiveness of various SDM interventions. ${ }^{51}$

\section{CONCLUSION}

Dermatologists and rheumatologists experience knowledge, skill and confidence gaps in relation to childbearing, treatment and management discussions with women of reproductive age with CID. They also experience challenges collaborating with other healthcare providers involved in the care of these patients. Some hold unfavourable attitudes towards SDM. Blendedlearning interventions, combining individual and teambased learning, may assist CID specialists in developing effective communication and patient engagement skills. Future studies may wish to investigate the perceived barriers and challenges from the perspective of patients, GPs and OB/GYNs.

\section{Author affiliations}

${ }^{1}$ Department of Research, AXDEV Group Inc, Brossard, Quebec, Canada ${ }^{2}$ Department of Dermatology, University of California, San Francisco, San Francisco, California, USA

${ }^{3}$ Dermatology, Palo Alto Medical Foundation, Mountain View, California, USA ${ }^{4}$ Policlinic of Rheumatology and Hiller Research Unit for Rheumatology, HeinrichHeine University Düsseldorf, Düsseldorf, Germany 
${ }^{5}$ Obstetric Medicine, Guy's and St Thomas' NHS Foundation Trust, London, UK ${ }^{6}$ Warwick Business School, University of Warwick, Warwick, UK

Acknowledgements The authors would like to acknowledge Cécile Ecoffet and Richard Dean Jenkins (employees of UCB Pharma) for critical input in the study conceptualisation, identification of relevant references and critical revision of the manuscript; Patrice Lazure (director of research, AXDEV Group) for ensuring quality of research conduct; Sara Labbé and Sacha Zahabi (researchers, AXDEV Group) for supporting data collection and coding of transcripts; Olivier Jacob and Randa Dalle (project managers, AXDEV Group) for supporting the process for recruitment of participants; Simone E Auteri and Christina Popova (employees of UCB Pharma) for critical revision of this manuscript. The authors would also like to thank all the participants who took part in this study.

Contributors SM conceptualised and designed the study. JEM, RF, CN-P and IV contributed to the refinement of the study design and methodologies. MA led the development of data collection tools, monitored the recruitment of participants and analysed both qualitative and quantitative data. MP supported research activities, including final analysis. SM, JEM, RF, CN-P and IV contributed to the interpretation of findings. MA and MP drafted the manuscript, which was critically reviewed by SM, JEM, RF, CN-P and IV for important intellectual content. All authors have approved of the final version of the manuscript and have agreed to be accountable for all aspects of the work.

This work was supported by UCB Pharma educational research funds.

Disclaimer The views expressed are those of the author(s) and not necessarily those of the NIHR or the Department of Health and Social Care.

Competing interests JEM has worked as a paid consultant to lead advisory boards and non-branded talks on the management of psoriasis in women for UCB BioPharma. RF has worked as a paid consultant for UCB BioPharma, and speaker for AbbVie, Amgen, Biogen, BMS, Celgene, Chugai, GSK, Janssen, Lilly, Medac, MSD, Novartis, Roche, UCB BioPharma and Pfizer. CN-P has worked as a paid consultant and speaker for UCB BioPharma. IV has worked as a paid consultant and speaker for UCB BioPharma and speaker for Novartis, Boehringer Ingelheim. IV was supported by the National Institute for Health Research (NIHR) Applied Research Collaboration (ARC) West Midlands. SM is the CEO and founder of AXDEV Group, AXDEV Global and AXDEV Europe. MA and MP are employees of AXDEV Group.

\section{Patient consent for publication Not required}

Ethics approval The study, including the investigational site, was approved by VERITAS IRB, an international independent ethical review board.

Provenance and peer review Not commissioned; externally peer reviewed.

Data availability statement Questions used in the interviews and survey can be found in online supplemental file 1 . The coding structure can be found in online supplemental file 2. Additional de-identified data can be shared on reasonable request for purposes of further study of this topic by researchers with a methodologically sound proposal until data are destroyed in correspondence with research ethics and privacy guidelines. Proposals should be directed to murrays@ axdevgroup.com. All researchers with qualified proposals will be required to sign a data access and confidentiality agreement.

Supplemental material This content has been supplied by the author(s). It has not been vetted by BMJ Publishing Group Limited (BMJ) and may not have been peer-reviewed. Any opinions or recommendations discussed are solely those of the author(s) and are not endorsed by BMJ. BMJ disclaims all liability and responsibility arising from any reliance placed on the content. Where the content includes any translated material, BMJ does not warrant the accuracy and reliability of the translations (including but not limited to local regulations, clinical guidelines, terminology, drug names and drug dosages), and is not responsible for any error and/or omissions arising from translation and adaptation or otherwise.

Open access This is an open access article distributed in accordance with the Creative Commons Attribution Non Commercial (CC BY-NC 4.0) license, which permits others to distribute, remix, adapt, build upon this work non-commercially, and license their derivative works on different terms, provided the original work is properly cited, appropriate credit is given, any changes made indicated, and the use is non-commercial. See: http://creativecommons.org/licenses/by-nc/4.0/.

\section{ORCID iDs}

Suzanne Murray http://orcid.org/0000-0002-3534-8379

Monica Augustyniak http://orcid.org/0000-0003-2825-5497

Ivo Vlaev http://orcid.org/0000-0002-3218-0144
REFERENCES

1 Nelson-Piercy C, Vlaev I, Harris K, et al. What factors could influence physicians' management of women of childbearing age with chronic inflammatory disease? A systematic review of behavioural determinants of clinical inertia. BMC Health Serv Res 2019;19:1-9.

2 Peterson EA, Lynton J, Bernard A, et al. Rheumatologic medication use during pregnancy. Obstet Gynecol 2020;135:1161-76.

3 Østensen M, Andreoli L, Brucato A, et al. State of the art: reproduction and pregnancy in rheumatic diseases. Autoimmun Rev 2015;14:376-86.

4 Smeele H, Dolhain R, Elsevier. Current perspectives on fertility. pregnancy and childbirth in patients with Rheumatoid Arthritis. in Seminars in arthritis and rheumatism 2019.

5 Götestam Skorpen C, Hoeltzenbein M, Tincani A, et al. The EULAR points to consider for use of antirheumatic drugs before pregnancy, and during pregnancy and lactation. Ann Rheum Dis 2016; 75:annrheumdis-2015-208840

6 Flint J, Panchal S, Hurrell A, et al. BSR and BHPR guideline on prescribing drugs in pregnancy and breastfeeding-Part I: standard and biologic disease modifying anti-rheumatic drugs and corticosteroids. Rheumatology 2016;55:1693-7.

7 Sammaritano LR, Bermas BL, Chakravarty EE, et al. 2020 American College of rheumatology guideline for the management of reproductive health in rheumatic and musculoskeletal diseases. Arthritis Care Res 2020;72:461-88.

8 Smith CH, Jabbar-Lopez ZK, Yiu ZZ, et al. British association of dermatologists guidelines for biologic therapy for psoriasis 2017. Br J Dermatol 2017;177:628-36.

9 Smith $\mathrm{CH}$, Yiu ZZN, Bale T, et al. British association of dermatologists guidelines for biologic therapy for psoriasis 2020: a rapid update. Br J Dermatol 2020;183:628-37.

10 Nast Aet al. EUROGUIDERM guideline for the systemic treatment of psoriasis vulgaris 2020 .

11 Mariette X, Förger F, Abraham B, et al. Lack of placental transfer of certolizumab pegol during pregnancy: results from CRIB, a prospective, postmarketing, pharmacokinetic study. Ann Rheum Dis 2018;77:228-33.

12 Clowse MEB, Scheuerle AE, Chambers C, et al. Pregnancy outcomes after exposure to certolizumab pegol: updated results from a pharmacovigilance safety database. Arthritis Rheumatol 2018:70:1399-407.

13 Julsgaard M, Christensen LA, Gibson PR, et al. Concentrations of Adalimumab and Infliximab in Mothers and Newborns, and Effects on Infection. Gastroenterology 2016;151:110-9.

14 Wexler R. Six steps of shared decision making. Informed Medical Decisions Foundation 2012:1-2.

15 Graffigna G, Barello S. Spotlight on the patient health engagement model (Phe model): a psychosocial theory to understand people's meaningful engagement in their own health care. Patient Prefer Adherence 2018;12:1261-71.

16 Rennke S, Yuan P, Monash B, et al. The SDM 3 circle model: a literature synthesis and adaptation for shared decision making in the hospital. J Hosp Med 2017;12:1001-8.

17 Taube K-M. Patient-Doctor relationship in dermatology: from compliance to concordance. Acta Derm Venereol 2016;96:25-9.

18 Beers E, Lee Nilsen M, Johnson JT. The role of patients: shared decision-making. Otolaryngol Clin North Am 2017;50:689-708.

19 The share approach, October 2020. Available: https://www.ahrq.gov/ health-literacy/professional-training/shared-decision/index.html

20 Elwyn G, Laitner S, Coulter A, et al. Implementing shared decision making in the NHS. BMJ 2010;341:c5146.

21 Mathijssen EGE, Vriezekolk JE, Popa CD, et al. Shared decision making in routine clinical care of patients with rheumatoid arthritis: an assessment of audio-recorded consultations. Ann Rheum Dis 2020;79:170-5.

22 van der Kraaij GE, Vermeulen FM, Smeets PMG, et al. The current extent of and need for shared decision making in atopic dermatitis and psoriasis in the Netherlands: an online survey study amongst patients and physicians. J Eur Acad Dermatol Venereol 2020;34:2574-2583.

23 Creswell JWet al. Best practices for mixed methods research in the health sciences. Bethesda (Maryland): National Institutes of Health, 2011 2013:541-5.

24 Moore DE. Needs assessment in the new health care environment: combining discrepancy analysis and outcomes to create more effective CME. J Contin Educ Health Prof 1998;18:133-41.

25 Turner SF, Cardinal LB, Burton RM. Research design for mixed methods: a triangulation-based framework and roadmap. Organizational Research Methods 2017;20:243-67.

26 Fusch PI, Ness LR. Are we there yet? data saturation in qualitative research. The qualitative report 2015;20:1408. 
27 Oyeyemi GM, Adewara AA, Adebola FB, et al. On the estimation of power and sample size in test of independence. Asian J. of Mathematics \& Statistics 2010;3:139-46.

28 O'Cathain A, Murphy E, Nicholl J. The quality of mixed methods studies in health services research. $J$ Health Serv Res Policy 2008;13:92-8.

29 Murray S, Awartani KA, Péloquin S. International challenges in patient-centred care in fertility clinics offering assisted reproductive technology: providers' gaps and attitudes towards addressing the patients' psychological needs. J Eur CME 2015;4:27578.

30 Murray S, McLintock C, Lazure P, et al. Needs and challenges among physicians and researchers in thrombosis and hemostasis: results from an international study. Res Pract Thromb Haemost 2019;3:626-38.

31 Michie S, Atkins L, West R. The behaviour change wheel.. In: A guide to designing interventions. 1st. Great Britain: Silverback Publishing, 2014: 1003-10.

32 West R, Michie S. A brief introduction to the COM-B Model of behaviour and the PRIME Theory of motivation [v1]. Qeios 2020.

33 Atkins L, Francis J, Islam R, et al. A guide to using the theoretical domains framework of behaviour change to investigate implementation problems. Implementation Science 2017;12:1-18.

34 . Interprofessional education collaborative expert panel, core competencies for interprofessional collaborative practice. Washington DC; 2011.

35 Thomas A, Kuper A, Chin-Yee B, et al. What is "shared" in shared decision-making? Philosophical perspectives, epistemic justice, and implications for health professions education. J Eval Clin Pract 2020;26:409-18.

36 ICC/ESOMAR. ICC/ESOMAR International Code on market, opinion and social research and data analytics, 2016. Available: https://www. esomar.org/what-we-do/code-guidelines

37 Tongco MDC. Purposive sampling as a tool for informant selection. Ethnobotany Research and Applications 2007;5:147-58.

38 DeJonckheere M, Vaughn LM. Semistructured interviewing in primary care research: a balance of relationship and rigour. Fam Med Community Health 2019;7:e000057.

39 Dominik Johannes L. Too fast, too straight, too Weird: non-reactive indicators for Meaningless data in Internet surveys. Survey Research Methods 2019;13.

40 Meade AW, Craig SB. Identifying careless responses in survey data. Psychol Methods 2012;17:437-55.
41 Fereday J, Muir-Cochrane E. Demonstrating rigor using thematic analysis: a hybrid approach of inductive and deductive coding and theme development. Int J Qual Methods 2006;5:80-92.

42 Joffe H, Yardley L. Content and thematic analysis. Research methods for clinical and health psychology 2004;56:68.

43 Hoover RS, Koerber AL. Using NVivo to answer the challenges of qualitative research in professional communication: benefits and best practices tutorial. IEEE Trans Prof Commun 2009;54:68-82.

44 McKight PE, Najab J. Kruskal-wallis test. The corsini encyclopedia of psychology 2010:1.

45 Birru Talabi M, Clowse MEB, Blalock SJ, et al. Perspectives of adult rheumatologists regarding family planning counseling and care: a qualitative study. Arthritis Care Res 2020;72:452-8.

46 Mathijssen EGet al. Exploring healthcare professionals' knowledge, attitudes and experiences of shared decision making in rheumatology. RMD open 2020;6.

47 Alameddine M, AlGurg R, Otaki F, et al. Physicians' perspective on shared decision-making in Dubai: a cross-sectional study. Hum Resour Health 2020;18:1-9.

48 Joseph-Williams N, Lloyd A, Edwards A, et al. Implementing shared decision making in the NHS: lessons from the magic programme. BMJ 2017;357:j1744-357.

49 Levounis P, Arnaout B, Marienfeld C. Motivational interviewing for clinical practice first edition ED. Arlington, Virginia: American Psychiatric Association Publishing, 2017.

50 Scholl I, LaRussa A, Hahlweg P, et al. Organizational- and systemlevel characteristics that influence implementation of shared decision-making and strategies to address them - a scoping review. Implement Sci 2018;13:40.

51 Coates D, Clerke T. Training interventions to equip health care professionals with shared decision-making skills: a systematic scoping review. J Contin Educ Health Prof 2020;40:100-19.

52 Kostick KM, Trejo M, Volk RJ, et al. Using Nudges to enhance clinicians' implementation of shared decision making with patient decision AIDS. MDM Policy Pract 2020;5:2381468320915906:23814 6832091590.

53 Taylor MJ, McNicholas C, Nicolay C, et al. Systematic review of the application of the plan-do-study-act method to improve quality in healthcare. BMJ Qual Saf 2014;23:290-8. 